\title{
The vasculature in sepsis: delivering poison or remedy to the brain?
}

\author{
Benjamin H. Singer \\ Department of Internal Medicine, Division of Pulmonary and Critical Care Medicine, University of Michigan Medical School, Ann Arbor, Michigan, USA.
}

\begin{abstract}
Survivors of sepsis and other forms of critical illness frequently experience significant and disabling cognitive and affective disorders. Inflammation, ischemia, and glial cell dysfunction contribute to this persistent brain injury. In this issue of the $J C I$, Hippensteel et al. show that endothelial injury in animal models of sepsis or endotoxemia leads to shedding of heparan fragments from the endothelial glycocalyx. These fragments directly sequester brain-derived neurotrophic factor and impair hippocampal longterm potentiation, an electrophysiologic correlate of memory. The authors further explore the specific characteristics of heparan fragments that bind neurotrophins and the presence of these fragments in the circulation of patients who survive sepsis. This study highlights an important mechanism by which vascular injury can impair brain function.
\end{abstract}

\section{Persistent brain injury in sepsis survivors}

Mortality from sepsis has fallen markedly with improvements in supportive care (1). Although over 14 million patients survive a hospitalization for sepsis each year, they are still not made whole by the life-saving care they receive (2). Rather, sepsis survivors frequently experience functional disability, along with increased risk of rehospitalization and death. Persistent brain dysfunction is a common and life-changing consequence of critical illness survivorship and results in both cognitive decline and a high incidence of affective disorders such as anxiety, depression, and posttraumatic stress $(3,4)$.

Multiple factors contribute to persistent brain dysfunction in sepsis survivors. Neuropathologic studies of sepsis in patients have largely been confined to those who succumb to their acute illness and have revealed that ischemia, hemorrhage, and white matter injury commonly occur at the extremes of critical illness (5). It is unclear, however, whether brain injury that results from the hypoperfusion, hypoxia, and circulatory dysfunction that underlie multiorgan dysfunction syndrome in sepsis is treatable or preventable by means other than improved supportive care of the underlying critical illness. Studies using animal models, therefore, have focused heavily on inflammatory mechanisms that extend beyond the period of critical illness and into the period of survivorship. Neuroinflammation, including activation of microglia and astrocytes, is also a prominent component of brain injury in sepsis $(6,7)$.

Survivors of endotoxemia, abdominal infection, or pneumonia experience multiple persistent inflammatory insults to the brain. Neutrophils and inflammatory monocytes traffic and invade the brain parenchyma (8). Expression of cytokines and alarmins in the brain persists for weeks following sepsis $(9,10)$. Microglia are especially susceptible to long-term reprogramming after high-dose endotoxin exposure (11), though the balance of tolerance and priming that results from persistent

Related Article: p. 1779

Conflict of interest: The author has declared that no conflict of interest exists.

exposure to proinflammatory signals over time is probably governed by a network of interactions among glia and neurons (12). In addition to the activation of intrinsic neuroinflammatory responses, sepsis may result in increased blood-brain barrier permeability and entry of inflammatory signals from the circulation $(13,14)$.

Despite evidence pointing to multiple mechanisms of persistent brain injury in sepsis survivors, the connection from a panoply of vascular and inflammatory insults to an actual persistent, reversible neuronal dysfunction in sepsis survivors remains obscure. Cytokine signaling and direct activation of innate immune receptors play an important role in the maintenance and elimination of synapses in both normal physiology and disease (15, 16 ), and these same mechanisms are presumably at work in the brains of sepsis survivors. Inhibition of IL-1 signaling and microglial activation rescues impairment of the long-term potentiation (LTP) that occurs acutely after sepsis or endotoxemia $(17,18)$. Though cytokines act directly on neurons and glia to change their intrinsic physiology and structure, inflammation may also activate a cascade of potentially reversible dysfunctions in the processing of neurotrophins and other signals important for circuit homeostasis. For example, brain-derived neurotrophic factor (BDNF) is an agonist of the TrkB receptor kinase that is required for long-lasting facilitation of synaptic transmission in LTP. In aged animals, systemic infection has been associated with prolonged increases in IL-1 $\beta$ expression and reductions in hippocampal BDNF, as well as sustained but reversible impairment of LTP (19).

\section{BDNF sequestration as a mechanism of learning and memory impairment}

In this issue of the JCI, Hippensteel and colleagues demonstrate that microvascular injury in sepsis results in impairment of learning and memory by a far more 


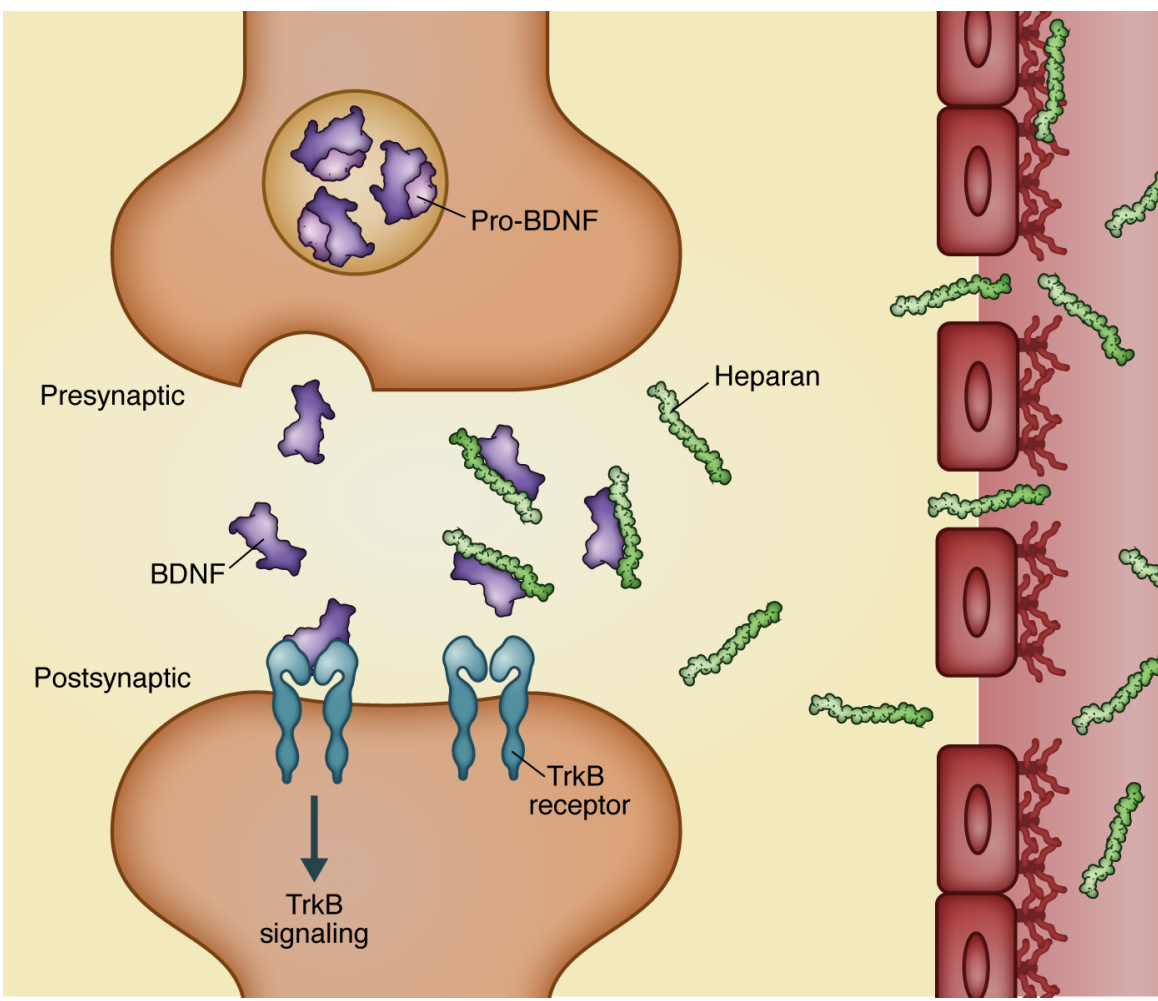

Figure 1. Injury to the endothelial glycocalyx in sepsis results in the release of heparan sulfate fragments into the brain parenchyma. Heparan sulfate fragments then bind and sequester BDNF, preventing activation of the TrkB receptor and leading to the loss of downstream TrkB signaling and protein synthesis that support synaptic strengthening during LTP. Illustrated by Rachel Davidowitz.

direct mechanism: sequestration of BDNF by products of endothelial glycocalyx injury (20). The endothelial glycocalyx is a meshwork of extracellular protein and carbohydrate molecules that supports endothelial function (21). Sepsis degrades the glycocalyx and leads to pathologic vascular permeability and leukocyte adhesion. Here, however, the authors found that heparan sulfate fragments, constituents of the glycocalyx itself, interfered with brain function. Circulating heparan fragments were elevated in patients with sepsis and were associated with cognitive impairment in sepsis survivors. Murine sepsis survivors demonstrated deficits in hippocampus-dependent behavior and LTP impairment. While total levels of hippocampal BDNF were not reduced after sepsis, sulfated heparan fragments functionally sequester BDNF (Figure 1). The authors were not able to specifically disrupt the interaction of heparan sulfate fragments and BDNF to restore LTP. However, supplementation of BDNF ex vivo reversed the electrophysiologic effect of endotoxemia, and direct TrkB agonism overcame the inhibitory effect of highly sulfated heparans on LTP.

\section{Endothelial injury products in future studies of brain injury}

The possibility that products of endothelial injury can interfere directly with brain function has several implications for future studies dealing with the prevention and treatment of brain injury associated with sepsis. The dominant model of persistent brain injury in sepsis ascribes neuronal dysfunction to a combination of circulatory, immune, and glial dysregulation. Cognitive dysfunction has been associated with vascular injury during sepsis, but this has usually been framed in terms of hemodynamic impairment or loss of barrier function $(14,22)$. The complex relationships among peripherally derived inflammatory mediators and leukocytes, microglia, and astrocytes in sepsis survivors that impinge on neuronal function depend on cascades of signaling pathways and changes in transcriptional programs that alter intrinsic neuronal function and expression of neurotrophins and physical- ly alter synaptic structure. Therefore, therapies should be directed either at preventing brain injury - by minimizing ischemia or improving cerebrovascular endothelial function - or at modulating the complex neuroimmune response that persists in sepsis survivors.

The findings of Hippensteel and colleagues do not refute the importance of ischemia and neuroinflammation, but do highlight a far more direct route by which endothelial injury can interfere in the function of neuronal networks. The authors made the striking observation that, since heparan sulfate fragments circulate, endothelial injury need not originate in the cerebral microcirculation to have a significant effect on brain function. Other groups have also recently found that products of endothelial injury, such as pulmonary endothelial amyloids produced in critically ill patients with pneumonia, enter the CSF and can impair LTP (23).

\section{Search for other components released in tissue injury}

If these neurotoxic products can originate anywhere in the body during critical illness that results in vascular injury, then what type of therapeutic strategies are needed to protect or restore function? While this study focuses on BDNF, we can speculate that glycocalyx fragments and extracellular matrix components released in tissue injury may bind to and inhibit other signals that are important for synaptic function. Preventing glycocalyx shedding entirely, given that patients with sepsis are already experiencing endothelial injury when they present for care, is not a practical strategy. Alternatively, a fuller understanding of which factors are bound by glycocalyx fragments would identify targets for pharmacologic interventions that might speed recovery from brain dysfunction in sepsis survivors. Finally, direct disruption of the physical sequestration of neurotrophins by heparan sulfate fragments would not only augment neurotrophin signaling, but also restore it in the very synapses where it is needed. Though the authors were not able to effectively reverse heparan sulfate fragment binding to BDNF in their study, their results suggest that this could be a powerful new direction for therapeutic research.

Persistent brain dysfunction in sepsis survivors is reflected in cognitive and 
affective disorders that cause significant disability, reduce quality of life, and contribute to morbidity and mortality. The mainstay of treating acute brain dysfunction has for many years been to alter neuromodulatory neurotransmission, mainly with typical or atypical antipsychotics. Recent landmark studies have demonstrated that this approach fails to improve brain function or alter short-term mortality $(24,25)$. While data on long-term outcomes are still forthcoming, the inability of antipsychotic medications to reverse or prevent delirium illustrates that simply masking behavior, such as agitation, will not restore the important homeostatic mechanisms that the brain needs to recover from acute injuries and prevent long-term dysfunction. We need a more nuanced understanding of the underlying pathophysiology of brain injury in survivors of critical illness, and here Hippensteel and colleagues have cast new light on previously unexplored but important mechanisms.

\section{Acknowledgments}

BHS is supported by the NIH (KO8NS101054) and the American Thoracic Society Foundation.

Address correspondence to: Benjamin $\mathrm{H}$. Singer, Division of Pulmonary and Critical Care Medicine, Department of Internal Medicine, University of Michigan Medical School, 4067 Biomedical Science Research Building, 109 Zina Pitcher Place, Ann Arbor, Michigan 48109, USA. Phone:734.764.4554; Email: singerb@med.umich.edu.
1. Kaukonen KM, Bailey M, Suzuki S, Pilcher D, Bellomo R. Mortality related to severe sepsis and septic shock among critically ill patients in Australia and New Zealand, 2000-2012. JAMA. 2014;311(13):1308-1316.

2. Prescott HC, Angus DC. Enhancing recovery from sepsis: a review. JAMA. 2018;319(1):62-75.

3. Iwashyna TJ, Ely EW, Smith DM, Langa KM. Long-term cognitive impairment and functional disability among survivors of severe sepsis. JAMA. 2010;304(16):1787-1794.

4. Rabiee A, et al. Depressive symptoms after critical illness: a systematic review and meta-analysis. Crit Care Med. 2016;44(9):1744-1753.

5. Sharshar T, Annane D, de la Grandmaison GL, Brouland JP, Hopkinson NS, Françoise G. The neuropathology of septic shock. Brain Pathol. 2004;14(1):21-33

6. Lemstra AW, et al. Microglia activation in sepsis: a case-control study. J Neuroinflammation. 2007;4:4

7. Denstaedt SJ, et al. S100A8/A9 drives neuroinflammatory priming and protects against anxiety-like behavior after sepsis. JImmunol. 2018;200(9):3188-3200.

8. Andonegui G, et al. Targeting inflammatory monocytes in sepsis-associated encephalopathy and long-term cognitive impairment. JCI Insight. 2018;3(9):99364

9. Semmler A, et al. Sepsis causes neuroinflammation and concomitant decrease of cerebral metabolism. J Neuroinflammation. 2008;5:38.

10. Chavan SS, et al. HMGB1 mediates cognitive impairment in sepsis survivors. Mol Med. 2012;18:930-937.

11. Wendeln AC, et al. Innate immune memory in the brain shapes neurological disease hallmarks. Nature. 2018;556(7701):332-338.

12. Chu $\mathrm{CH}$, et al. Neurons and astroglia govern microglial endotoxin tolerance through macrophage colony-stimulating factor receptormediated ERK1/2 signals. Brain Behav Immun. 2016;55:260-272

13. Danielski LG, et al. Brain barrier breakdown as a cause and consequence of neuroinflammation in sepsis. Mol Neurobiol. 2018;55(2):1045-1053.

14. Hughes CG, et al. Relationships between markers of neurologic and endothelial injury during critical illness and long-term cognitive impairment and disability. Intensive Care Med. 2018;44(3):345-355.

15. Stellwagen D, Malenka RC. Synaptic scaling mediated by glial TNF- $\alpha$. Nature. 2006;440(7087):1054-1059.

16. Garré JM, Silva HM, Lafaille JJ, Yang G. CX3CR1+ monocytes modulate learning and learning-dependent dendritic spine remodeling via TNF- $\alpha$. Nat Med. 2017;23(6):714-722.

17. Hoshino K, Hayakawa M, Morimoto Y. Minocycline prevents the impairment of hippocampal long-term potentiation in the septic mouse. Shock. 2017;48(2):209-214.

18. Skelly DT, et al. Acute transient cognitive dysfunction and acute brain injury induced by systemic inflammation occur by dissociable IL-1-dependent mechanisms [published online ahead of print June 6, 2018]. Mol Psychiatry. https://doi.org/10.1038/s41380-018-0075-8.

19. Tanaka N, Cortese GP, Barrientos RM, Maier SF, Patterson SL. Aging and an immune challenge interact to produce prolonged, but not permanent, reductions in hippocampal L-LTP and $\mathrm{mBDNF}$ in a rodent model with features of delirium. eNeuro. 2018;5(3):ENEURO.0009-18.2018.

20. Hippensteel JA, et al. Circulating heparan sulfate fragments mediate septic cognitive dysfunction. J Clin Invest. 2019;129(4):1779-1784.

21. Uchimido R, Schmidt EP, Shapiro NI. The glycocalyx: a novel diagnostic and therapeutic target in sepsis. Crit Care. 2019;23(1):16.

22. Hughes CG, et al. Association between endothelial dysfunction and acute brain dysfunction during critical illness. Anesthesiology. 2013;118(3):631-639.

23. Lin MT, et al. Nosocomial pneumonia elicits an endothelial proteinopathy: evidence for a source of neurotoxic amyloids in critically ill patients [published online ahead of print October 3 , 2018]. Am J Respir Crit Care Med. https://doi. org/10.1164/rccm.201801-0060LE.

24. Girard TD, et al. Haloperidol and Ziprasidone for treatment of delirium in critical illness. $N$ EnglJ Med. 2018;379(26):2506-2516.

25 . van den Boogaard M, et al. Effect of haloperidol on survival among critically ill adults with a high risk of delirium. JAMA. 2018;319(7):680-690. 